\title{
Optimization of Resource Mining in Distributive Sharing (ORMDS) Scheme for Future Heterogeneous Network
}

\author{
Satyanarayan K. Padaganur \\ Department of Electronics \& Communication Engineering \\ B.L.D.E.A's Dr. P.G.H CET, Vijayapur-586103, India. \\ E-mail: padaganursk@gmail.com
}

Jayashree D. Mallapur

Department of Electronics and communication, Basaveshwar Engineering College, Bagalkot, India

E-mail: bdmallapur@yahoo.co.in

Received: 09 March 2018; Accepted: 07 July 2018; Published: 08 August 2018

\begin{abstract}
In future heterogeneous network communication systems, driven by the evolution of today's most demanding applications, resource allocation and its mining will play an increasingly significant role in our daily life for different use of applications facing a rapid growth in data traffic demands recently. In this work based on future network architecture, we proposed a scheme called Optimization of Resource Mining in Distributive Sharing (ORMDS) for better utilization of in-house residual buffer to improve performance of the network in-terms of maximizing the efficiency, minimizing network delay, call drop and buffer mining. A distributed buffer allocation and mining in the framework is proposed to facilitate different multimedia application in future network. Resource is measured and updated in the buffer table of router. Buffer table is updated based on execution of optimization computation model, which uses optimization seeking model for determining the value of objective function. This minimum value of objective function will improve the resource utilization of the network. The proposed scheme reduces the mining time of the buffer in sharing system and optimizes the delay by using distributed sharing option. This ultimately gives optimum resource sharing at a different instant of time.
\end{abstract}

Index Terms-Resource mining, Optimization, Buffer allocation and Future heterogeneous network.

\section{INTRODUCTION}

The future heterogeneous networks believe in resource sharing as the resource availability has become a bottle neck. The requirement of resources such as bandwidth and buffer in digital communication exists in kilobytes to gigabytes. Hence there is a maximum request for resources on dynamic availability of these in the network. In the network, the buffer sharing is not dynamic but static. That is, it is shared between two restricted members who are earlier registered and notified and if the buffer is not available, then the real time application request is denied which will lead to the maximum call drop in the network. The 4G LTE network or next generation network of wireless technologies will provide support to higher data transfer rate, larger system capacity, next generation Internet support, seamless services, global roaming and flexible network architectures. To reach these several goals, the network of the future must be able to provide support for heterogeneous networking with support for both horizontal and vertical handovers (ex. WLAN to cellular and vice versa), seamless roaming and mobile IP.

There are two application supported by future generation network like time-critical and non-time critical applications. Non-time critical applications sustain static allocations and sharing. Whereas dynamic applications experience high rate of failure, hence real time applications needs are temporarily, but priority based sharing scheme. To support real time applications, we need optimized load balanced resource sharing system. The optimization load balancing is aimed at to provide required bandwidth and buffer at the time of execution of real time application. The optimization techniques always help to efficient usage of resources at QoS maintenance. Now days, the optimization technique software embedded for wireless technology will lead better Qos maintenance than the other existing techniques. In our scheme, we have planning to have segmented dynamic resource sharing along with the optimized neighbors to reduce delay and retrieving time. In this work, we are using router level optimized distributive sharing scheme. This will maximize throughput, efficiency and minimize buffer mining delay and call drop. 


\section{RELATED WORK}

The work presented in [1], proposes an algorithm for optimized balancing of load in a system of cloud and the results shows that the importance of the sharing. [2] This paper presents about various load balancing schemes in cloud environment. [3] This paper describe about different load sharing approaches for gateway level and router level of working. It explains about the load balancing of the network. [4] It tells about different techniques of persistent methods. This paper provides that persistence weighted load blanching procedure give better performance than others. [5] It represents the heuristic for computation pdf of queue in poison arrival with the acceptable system performance level. [6] This paper explains about how to reduce the rejection rate in heterogeneous environment compared to conventional methods, which will help to improve the system efficiency. [7] This paper presents about how packet service is implemented for heterogeneous wireless network using load balancing approach, which will greatly reduce the blocking probabilities. [8] This paper focus on 3GPP heterogeneous network. Using dynamic load-balancing and sub-optimal schemes, it improves the throughput of the system. [9] This paper proposes about a scalable, effective and reliable approach for allocating and balancing the amount of work between the scattered resources available on Grid systems. The work implemented approach is self-organized, scalable, and robust rest on local data for load distribution. [10] This paper describe about how load balancer will implemented in software defined network for improving performance of the system in terms of minimum response time. [11] This paper explained about, how load balancing scheme is used to avoid the congestion for improving the efficiency. The planned scheme is also effective about energy consumption and dependable about data envelope communication. [12] In this, it proposes a new load balancing method using neural networks.to handle admission control in new service and heavily loaded network. The equation and optimization seeking methods can be used from [13]. It clearly explains how objective function can be optimized utilizing Linear or Non-linear programming scheme. This paper present about, how buffer awareness will improve the uplink transmission in LTE network. This will lead to increase in network efficiency. [15] This paper express about, how new admission control and heavy load traffic can overcome by using load balancing algorithm using neural network. [16] This paper focus on hybrid approach used for resource assignment for scheduling. This also use optimization scheme with ant colony and round robin techniques. [17] This paper focus on different efficient energy scheme for dense network. Proposed work is intended designed for proper utilization of energy in the network.

\section{PROPOSED WORK}

The main contribution of this proposed work is to utilize in-house resources in an efficient manner. It is always difficult to provide continuous resources for a real time multimedia application with minimum bandwidth availability. So, by using in-house buffer from the neighbor nodes, we provide better QoS to the users in highly loaded network, which is shown in Fig.1.

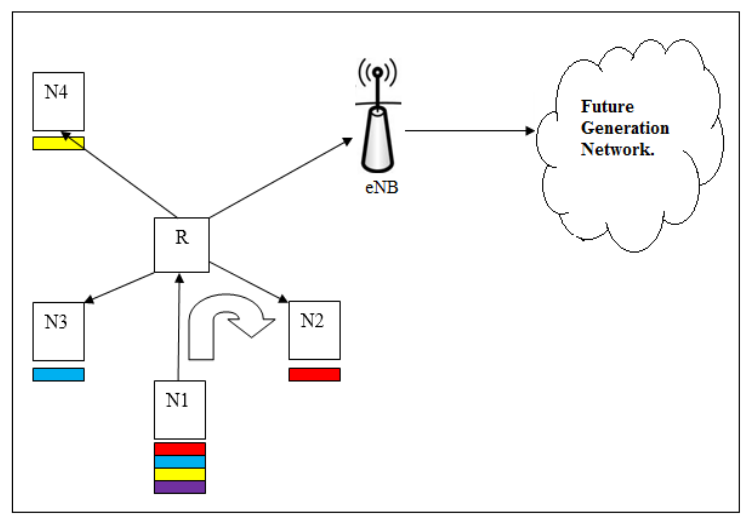

Fig.1. Scheme of Execution

With the current technique it becomes important that, some of the connected nodes to the access point had enough buffers available for the extra chunks to be saved. The current technique also assumes that some of the nodes have got lower capacity and links speed requirement such that part of their data exchange can handle the buffer allocation and mining (retrieval). In a critical data sharing session such as voice or video calls, it leads to a call drop. Once the network is again capable of handling the high speed data transmission and overloaded traffic it again switches back the traffic within the current network. Therefore by executing our scheme Optimization of Resource Mining in Distributive Sharing (ORMDS), we ensure a node is always able to transmit the data with its desired speed without having to wait for data rate change or network traffic congestion dependency. Therefore we call our proposed method as intelligent buffer allocation because the system can identified the constraints with the buffer mining and at the same time also knows when such an optimization is possible and when it not possible. In case when the system optimization is not possible the system offers traditional technique.

In this proposed work, we propose and developed a scheme called Optimization of Resource Mining in Distributive Sharing (ORMDS) scheme. The aim of the proposed scheme is to reduce buffer mining delay. Our proposed work will enhance the performance of the network in terms of maximizing the efficiency, minimizing mining delay and reduction in call drop. 


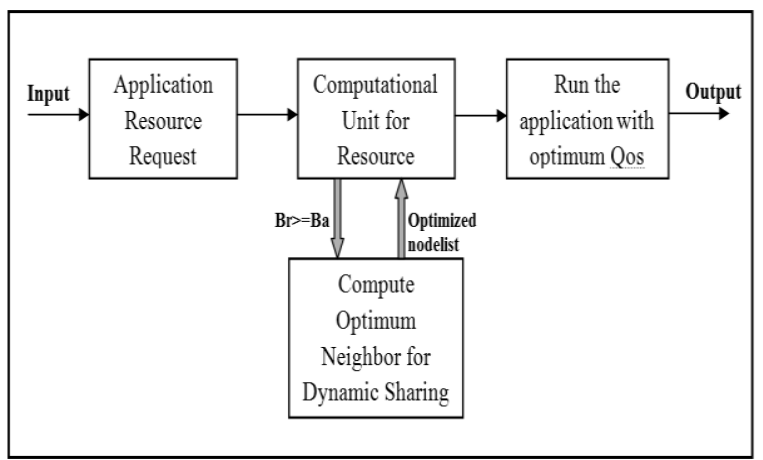

Fig.2. Block diagram of proposed work

Above Fig. 2 represent the proposed work block diagram. In heterogeneous network the application resource requirement changes are very uncertain and calculating resource dynamically is very challenging and competitive. In our scheme whenever application request arrives we compute resource availability. When resources are not sufficient to run the application we are planning to share segmented resources.

In segmented resource sharing, the resources are request from the neighbors. The neighbor will answer the segmented resources available with them. But sharing with all the neighbors become hectic, when recovering, hence we compute segmented resource sharing based on optimum technique.

The optimum neighbor list will be checked for minimum buffer mining delay with efficient sharing. The new list of neighbor is computing by running optimized algorithm with Non-Linear programming. These nodes will be allowed to share segmented resources. Once the request resource becomes available the remains nodes will not asked to share. Hence QoS challenges are met with reduction in call drop and increase in throughput.

\section{MATHEMATICAL MODEL}

From the mathematical model it is clear that the objective of the network is to offer a node not only for a seamless connectivity but also a mechanism by means of which buffer overflow in network packet loss have been reduced. There have been several past works which works with load balancing technique. A load balancing mechanism is one by means of which the overall load to be transmitted by node is divided into several chunks, and these chunks are transmitted into several different paths and generated by each of these individual nodes.

- Let 'n' represents the total mobile nodes.

- Let ' $\mathrm{m}$ ' represents the total registered neighbor nodes.

- Let ' $\mathrm{k}$ ' are the optimum neighbor nodes list.

- Initialize $\mathrm{i}=0, \mathrm{j}=0, \mathrm{k}=0$

1 For $\mathrm{i}=1$ to $\mathrm{n}$

$2 \mathrm{P}[\mathrm{i}]=\left\{\max\right.$ buffer $\mathrm{B}_{\mathrm{f}}$ AND [min $\mathrm{n}(\mathrm{i})$ delay OR $\mathrm{n}[\mathrm{i}]$ distance]\}

3 increment $\mathrm{k}$ till list is completed.

4 For $\mathrm{j}=1$ to $\mathrm{m}$

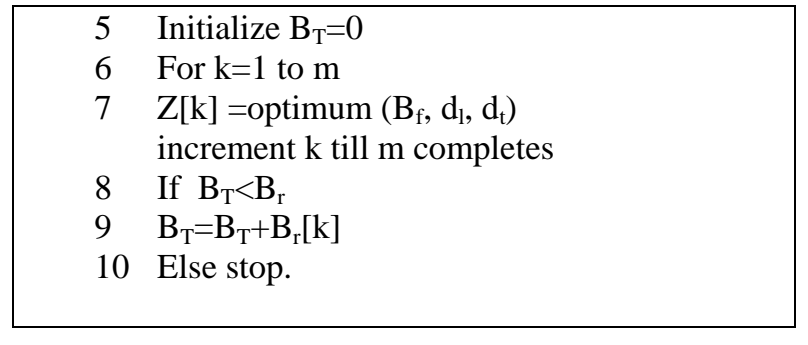

\section{AlgORITHM}

In this proposed work, three algorithms are designed and developed for optimal resource distribution.

First algorithm is developed for main proposed work i.e. to generation of heterogeneous model with all components of networks. If application arrives then it checks for buffer availability. For insufficient buffer it execute algorithm 2 .

The second algorithm is designed and developed for checking the buffer availability from the neighbor nodes. Initially nodes should register for load distribution.

The final algorithm will illustrate the optimal buffer distribution in the network. The objective function is computed based on buffer availability and distance of the nodes. For computation purpose it utilizes the GAMS Non-Linear programming scheme.

(Nomenclature: $n=$ number of nodes, $B_{r}=$ requested bandwidth from source node, $\mathrm{B}_{\mathrm{a}}=$ available sharing bandwidth of $i^{\text {th }}$ node.)

In this algorithm, the whole heterogeneous network topology with a different access points and different nodes are considered. Each different application is measured for their resource request. Whenever resource request comes from the application the buffer availability is checked if not sufficient algorithm 2 executed, in turn algorithm 3 is also executed.

\subsection{Algorithm 1: Main Program.}

\{Nomenclature: $\mathrm{n}=$ total number ofnodes, $\mathrm{m}=$ number of neighbor nodes, $\mathrm{z}=$ total number of optimized nodes, Bmax=Maximum buffer available, $\mathrm{Br}=\mathrm{Buffer}$ request, $\mathrm{Ba}=\mathrm{Buffer}$ available $\}$

Begin:

Step 1 Create network of with number of m nodes, Router and eNB.

Step 2 Initialize each node with resource.

Step 3 Check for buffer request.

Step 4 Call Algorithm 2

Step 5 Run the application to forward incoming data packets.

Step 6 go to Step 3 


\subsection{Algorithm 2: Neighbor List for resource availability.}

\{Nomenclature: $n=$ number of neighbor nodes, $\mathrm{Br}=$ Buffer request, $\mathrm{Ba}=$ Buffer available. $\}$

Begin

Step 1 Initialize each node with buffer availability

Step 2 Check for registered neighbor nodes.

Step 3 Call Algorithm 3

Step 4 Update buffer availability

Step 5 Refresh parameters

Step 6 Return buffer available

End.

4.3 Algorithm 3: Optimum list of neighbors with buffer availability.

\{Nomenclature: $\mathrm{Br}=\mathrm{Buffer}$ request, $\mathrm{Ba}=\mathrm{Buffer}$ available, bf[i]lower bound < bf[i] < bf[i] upper bound, dl[i]lower bound $<\mathrm{dl}[\mathrm{i}]<\mathrm{dl}[\mathrm{i}]$ upper bound, $\mathrm{dt}[\mathrm{i}]$ lower bound $<\mathrm{dt}[\mathrm{i}]<\mathrm{dt}[\mathrm{i}]$ upper bound

Where $\quad b f[i]=$ buffer availability of $i^{\text {th }}$ node,

$\mathrm{dl}[\mathrm{i}]=$ delay from $\mathrm{i}^{\text {th }}$ node,

$\mathrm{dt}[\mathrm{i}]=$ distance from $\mathrm{i}^{\text {th }}$ node, $\}$

Begin

Step 1 Initialize list of neighbor nodes.

Step 2 Z $[\mathrm{i}]=[\max (\mathrm{bf}) \quad$ AND $\min (\mathrm{dl}$ OR dt)]

Step 3 Apply GAMS NLP scheme.

Step 4 Where $\mathrm{Z}=$ optimum $\{\mathrm{bf}, \mathrm{dl}, \mathrm{dt}\}$

Step 5 if $\mathrm{Br}=\min \{\mathrm{Z}[\mathrm{i}]\}$

Step 6 Update optimum list

Step 7 List down best optimum neighbor nodes.

End.

Step 8 return Z[i]

\section{RESULTS AND DISCUSSIONS}

This section describes about performance parameters and results. This scheme is used to calculate the resource (buffer) mining delay and throughput of the network. It describes the outcomes of planned work and also relates with the static scheme. The work is simulated on i3 laptop using $\mathrm{C}$ programing and GAMS (General Algebraic Modeling System) software for optimization.

The factors of performance measured are mentioned below.

- Buffer mining time: Ihis is a time measurment for retrieval of segmented resources among the optimum neighbor list.

- Optimum delay: It is the time length of the process of application execution with our scheme.
- Distributive sharing: It is percentage of nodes which are decided with our scheme to share the resources.

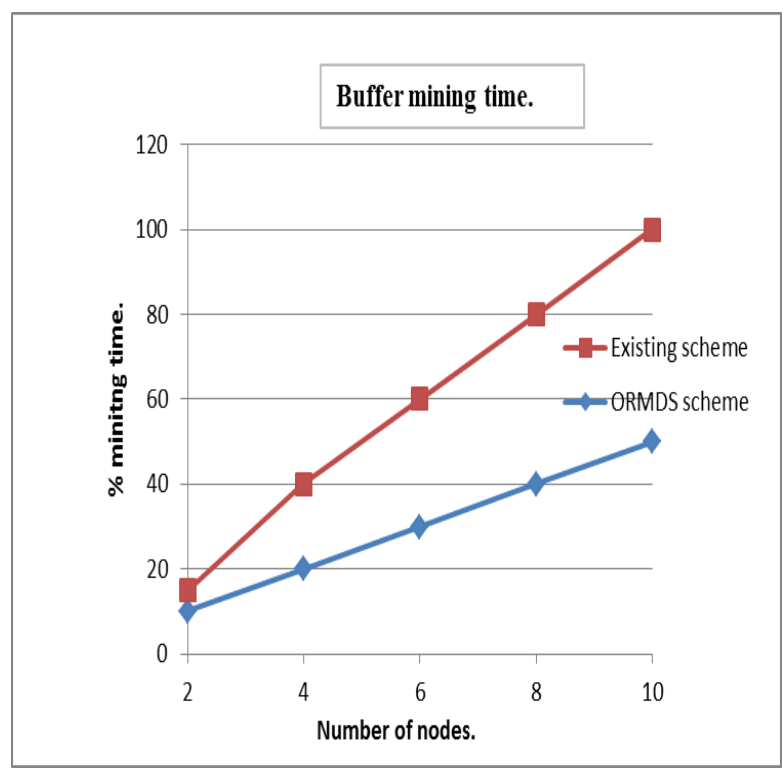

Fig.3. Buffer mining time v/s number of nodes.

The above result clearly gives 20 to $40 \%$ less in buffer mining time compared to the schemes without optimization as shown in Fig. 3. The optimum scheme enables us to restrict a distribution of segmented resources within a few registered neighbor nodes. Hence mining time also gets restricted to optimum list.

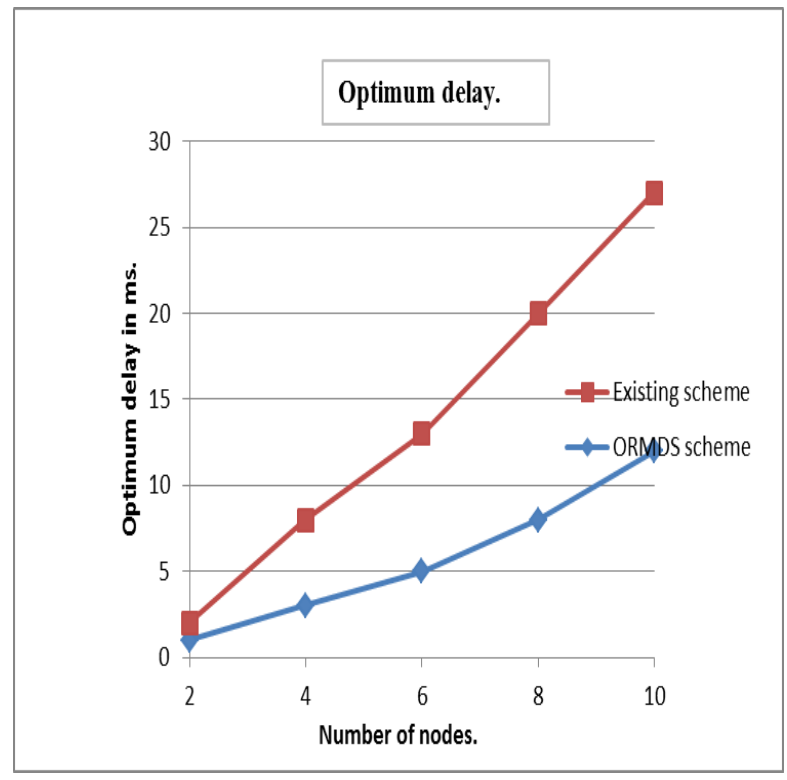

Fig.4. Optimum delay v/s number of nodes.

The optimum delay is a measurment of resource retrival and execution. When list is optimum the delay time gets reduced as shown in above results. The Fig. 4. Illustrates reduction in the above results $15-20 \%$ less in optimum delay compared to other non-optimum schemes. 


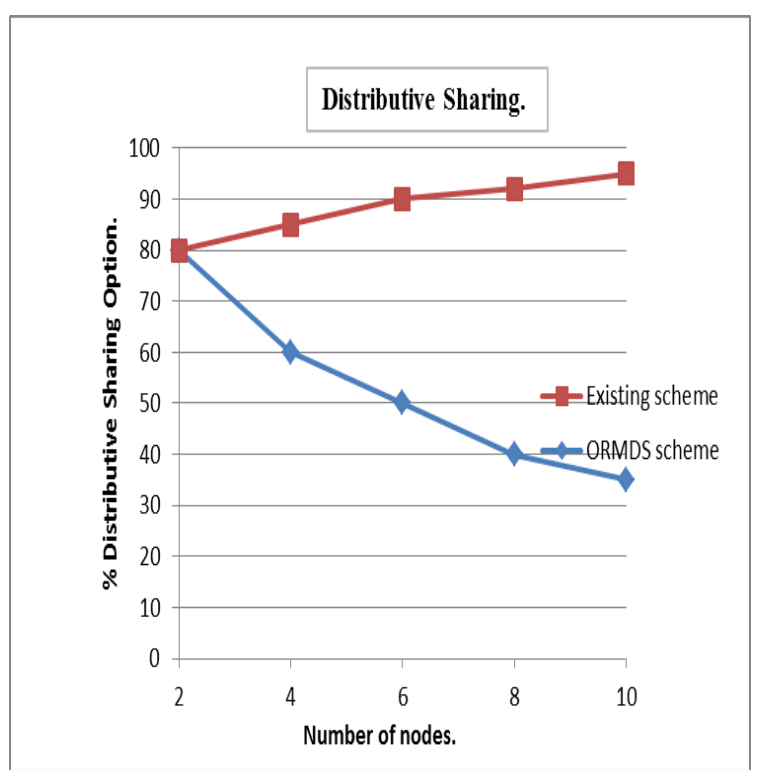

Fig.5. Distributed sharing v/s number of nodes.

The Fig. 5 represents the overall of distributive sharing parameter. Distributive system utilizes less number of nodes for buffer allocation and retrival. So it leads to minimum buffer mining time and maximum throughput. Intern it will improve the performance of network.

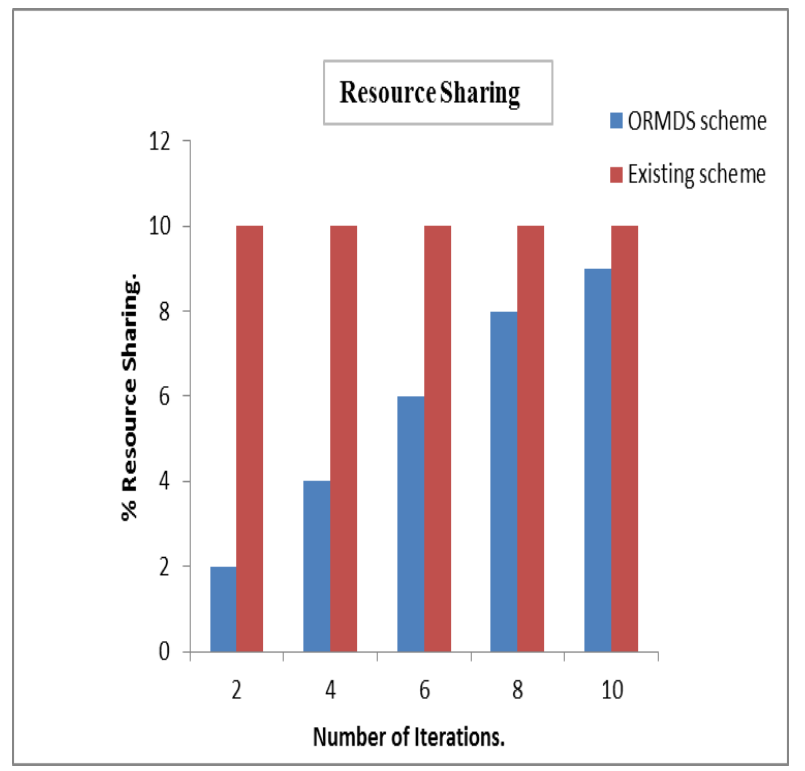

Fig.6. Resource sharing v/s number of iterations.

The above Fig. 6 describes about resource sharing of the proposed work. This diagram gives that utilization of resource sharing with optimization is better than without optimization. So it leads to minimizing delay and buffer mining time. Which are main performance parameters measured in this work.

\section{CONCLUSION}

Future data networks are the active area of research. With the growing rate of wireless users in future generation, managing the load and utilization of resources are great concern. The goal of this paper is to propose and develop an ORMDS scheme which uses the distributed sharing or parallel processing is a new trend in heterogeneous network. The distributed sharing will always reduce the burden on only neighbor but distributes the burden among the neighbors. Which will increases the speed of operation and reduces the call drops. In our scheme, we have gone for one step further by sharing the load with optimum list of neighbor nodes. The neighbors are checked for their optimum sharing capacity and then share. This reduces the data mining time in terms reduced delay and increased efficiency.

\section{REFERENCES}

[1] Ranesh Kumar Naha, and Mohamed Othman: Optimized Load Balancing for Efficient Resource Provisioning in the Cloud[S] IEEE 2nd International Symposium on Telecommunication Technologies (ISTT), Langkawi, Malaysia 2014)

[2] A.S. Milani, N.J.Navimipour: Load balancing mechanisms and techniques in the cloud environments: Systematic literature review and future trends[J]. Elsevier, Journal of Network and computer application, 2016

[3] Banani Das, Sudipta Roy:Load Balancing Techniques for Wireless Mesh Networks: A Survey[R]. IEEE Int. Symposium on Computational and Business Intelligence, 2013

[4] Krishnanjali A.Magade, Abhijit Patankar:Techniques for Load Balancing in Wireless LAN's[C]. IEEE Int. Con. on Communication and Signal Processing, April, 2014

[5] Dibyajyoti Guha, S.S. Pathak:Load Balancing using Past Information of Queue[C]. IEEE Int. Conf. 2011.

[6] Shiao-Li Tsao, et al:A Dynamic Load-Balancing Scheme for Heterogeneous Wireless Networks[C]. IEEE WCNC'14 Track 3 (Mobile and Wireless Networks), 2014.

[7] Chen Yuan-yuan, Qi Bing, Luo Yi-ting, Tang Liang-rui, : A Hybrid Dynamic Load Balancing Algorithm in Heterogeneous Wireless Packet Networks[C]. IEEE Int. Con. Fuzzy Systems and Knowledge Discovery (FSKD), 2012.

[8] Zhixiong Ding, Xingjun Wang and Wenming Yang:A Dynamic Load Balancing Algorithm in Heterogeneous Network[C]. IEEE 7th Int. Conf. on Intelligent Systems, Modelling and Simulation, 2016

[9] O. A. Rahmeh and P. Johnson:A Load Balancing Scheme for Latency Optimization in Grid Networks[C] IEEE Int. Conf. 2010.

[10] A M Koushika, S Thamarai Selvi:Load Balancing Using Software Defined Networking In Cloud Environment[C] IEEE, Int. Conf. on Recent Trends in Information Technology, 2014

[11] Minho Choi, Junhyung Kim, Sooyeol Yang, Namkoo Ha, and Kijun Han:Load Balancing for Efficient Routing in Wireless Sensor Networks[C], IEEE IMSCCS.2008.42.

[12] Xin Song1, Liangming Wu2, Xin Ren1, and Jing Gao1 :Load Balancing Algorithm Based on Neural Network in Heterogeneous Wireless Networks[M] Springer, LNCS 9377, pp. 463-472, 2015.

[13] Averill Law and David Kelton : Simulation Modeling and Analysis[M]. TMH $3^{\text {rd }}$ edition, 2003.

[14] Chiapin Wang and Xiagring Li: A Buffer-Aware Resource Allocation Scheme for 4G LTE Systems[S]. 2013 IEEE 17th International Symposium on Consumer Electronics (ISCE). 
[15] Xin Song et al: Load Balancing Algorithm Based on Neural Network in Heterogeneous Wireless Networks[M]. Springer LNCS 9377, pp. 463-472, 2015.

[16] Anuj Aggarwal, Rajesh Verma, Ajit Singh :An Efficient Approach for Resource Allocations using Hybrid Scheduling and Optimization in Distributed System", International Journal of Education and Management Engineering(IJEME), Vol.8, No.3, pp.33-42, 2018.DOI: 10.5815/ijeme.2018.03.04

[17] Sivaraj C, Alphonse P J A, Janakiraman T N,:Energyefficient and Load Distributed Clustering Algorithm for Dense Wireless Sensor Networks[J]. International Journal of Intelligent Systems and Applications (IJISA), Vol.9, No.5, pp.34-42, 2017. DOI: 10.5815/ijisa.2017.05.05

\section{Authors' Profiles}

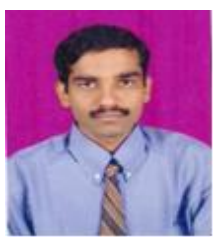

Mr. Satyanarayan. K. Padaganur: Obtained his B.E (E\&C) from Karnataka University, Dharwad, and M.Tech. (Digital Communication) from Visvesvaraya Technological University, Belagavi. He is working as Asst. Professor in the department of Electronics and Communication Engineering, B.L.D.E.A'S V.P.DR. P.G.Halakatti College of Engg. and Tech., Vijayapur (Karnataka), India. He has total 16 years of experience. His area of interest are Wireless communication, Wireless network, Computer network, Multimedia communication and Advance computer network. Currently he is pursuing Ph.D under Visvesvaraya Technological University, Belagavi.

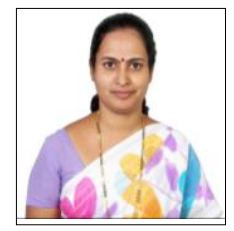

Dr. Jayashree. D. Mallapur: She is a professor in the department of Electronics and Communication Engineering, Basaveshwar Engineering College, Bagalkot, Karnataka, India. She has received B.E from Karnataka University, Dharwad, M.E from Gulbarga University and Doctorate from Visvesvaraya Technological University, Belagavi. She has 25 years of experience. Her area of interest is Wireless communication, Wireless network, fuzzy logic and multimedia communication. She has published 30 papers in National, International conferences and Journals. She is the life member of profession bodies like IEEE, ISTE, IEI and ETE.

How to cite this paper: Satyanarayan K. Padaganur, Jayashree D. Mallapur, "Optimization of Resource Mining in Distributive Sharing (ORMDS) Scheme for Future Heterogeneous Network", International Journal of Information Technology and Computer Science(IJITCS), Vol.10, No.8, pp.18-23, 2018. DOI: 10.5815/ijitcs.2018.08.03 